4 Bangalore S, Kamalakkannan G, Parkar S, et al. Fixed-dose combinations improve medication compliance: a meta-analysis. Am J Med 2007; 120: 713-719.

5 Gallardo CR, Rigau Comas D, Valderrama Rodríguez A, et al. Fixed-dose combinations of drugs versus singledrug formulations for treating pulmonary tuberculosis. Cochrane Database Syst Rev 2016; 5: CD009913.

6 Pasipanodya JG, Srivastava S, Gumbo T. Meta-analysis of clinical studies supports the pharmacokinetic variability hypothesis for acquired drug resistance and failure of antituberculosis therapy. Clin Infect Dis 2012; 55: 169-177.

7 Goutelle S, Bourguignon L, Maire P, et al. The case for using higher doses of first line anti-tuberculosis drugs to optimize efficacy. Curr Pharm Des 2014; 20: 6191-6206.

8 Zuur MA, Bolhuis MS, Anthony R, et al. Current status and opportunities for therapeutic drug monitoring in the treatment of tuberculosis. Expert Opin Drug Metab Toxicol 2016; 12: 509-521.

9 Sun Q, Zhang Q, Gu J, et al. Prevalence, risk factors, management, and treatment outcomes of first-line antituberculous drug-induced liver injury: a prospective cohort study. Pharmacoepidemiol Drug Saf 2016; 25: 908-917.

10 van der Burgt EP, Sturkenboom MG, Bolhuis MS, et al. End TB with precision treatment! Eur Respir J 2016; 47: $680-682$.

11 Ghimire S, Bolhuis MS, Sturkenboom MG, et al. Incorporating therapeutic drug monitoring into the World Health Organization hierarchy of tuberculosis diagnostics. Eur Respir J 2016; 47: 1867-1869.

12 Sotgiu G, Alffenaar JW, Centis R, et al. Therapeutic drug monitoring: how to improve drug dosage and patient safety in tuberculosis treatment. Int J Infect Dis 2015; 32: 101-104.

13 Pasipanodya JG, McIlleron H, Burger A, et al. Serum drug concentrations predictive of pulmonary tuberculosis outcomes. J Infect Dis 2013; 208: 1464-1473.

14 Pasipanodya JG, Gumbo T. Clinical and toxicodynamic evidence that high-dose pyrazinamide is not more hepatotoxic than the low doses currently used. Antimicrob Agents Chemother 2010; 54: 2847-2854.

15 Lei X, Huang K, Liu Q, et al. Are tuberculosis patients adherent to prescribed treatments in China? Results of a prospective cohort study. Infect Dis Poverty 2016; 5: 38.

\title{
Tuberculosis in London: the convergence of clinical and social complexity
}

\section{To the Editor:}

In large European cities, the tuberculosis (TB) epidemic is characteristically concentrated in vulnerable and under-served populations [1]. London has the highest number and annual incidence of TB in Europe and implemented routine surveillance on homelessness, drug and alcohol misuse and imprisonment among TB patients in 2009 [2]. This paper describes the clinical, public health and epidemiological characteristics of $\mathrm{TB}$ cases and the public health impact of social risk factors including risk of infectiousness, onward transmission, poor treatment adherence and drug resistance.

We analysed a cohort of adult London TB patients (2009-2012) including clinical and laboratory surveillance information. This was improved by matching against the Find\&Treat team's database, who support TB patients across London with complex social needs [3]. Homelessness, imprisonment, drug and alcohol misuse were defined as per national guidance [4]. Multi-drug resistant (MDR) TB was defined as per the World Health Organization (WHO), and poor treatment outcome was defined as not completing treatment within 12 months for rifampicin-sensitive patients, or within 24 months for rifampicin-resistant patients [5]. Recent migrants were defined as entering the UK less than 2 years before diagnosis. United Nations world region of birth was amended to a TB surveillance classification. Ethical approval was not required as this study was based on routine surveillance data held by Public Health England. Public Health England has Health Research Authority approval to hold and analyse national surveillance data for public health purposes.

Risk factors were identified for smear-positive pulmonary disease; isoniazid and MDR (restricted to culture-confirmed cases); non-adherence to treatment; and poor treatment outcomes (restricted to individuals notified 2009-2011). Univariable analysis generated odds ratios, with 95\% confidence intervals and Chi-squared test for significance. Multivariable logistic regression was used to generate adjusted odds ratios, built using likelihood ratio tests. Variables were retained in the final model if they improved the fit of the model $(\mathrm{p}<0.05)$ or confounded a different exposure. Potential interactions were investigated based on a priori knowledge. Data were analysed using Stata 12 (StataCorp LP, College Station, TX, USA).

Of the cohort of 12908 adult TB cases, 1321 (10\%) had one or more social risk factor: homelessness (550 (4\%)), imprisonment (349 (3\%)), drug (436 (3\%)) or alcohol misuse $(581(5 \%))$. Cases with social risk factors were more often male $(79 \%$ versus $55 \%$; $\mathrm{p}<0.001)$ UK born $(29 \%$ versus $12 \%$; $\mathrm{p}<0.001)$ white $(25 \%$ versus $9 \% ; \mathrm{p}<0.001)$ or black Caribbean $(7 \%$ versus $3 \% ; \mathrm{p}<0.001)$. Multiple factors were common (393 patients, 30\% reported two or more). 
We stratified the multivariable analysis for infectious disease by drug use due to the interaction between drug use and homelessness (likelihood ratio test, $\mathrm{p}=0.0071$ ). No further interactions were identified.

Among 4501 pulmonary patients with no history of drug use, 134 (58\%) out of 231 homeless patients were smear positive. Homelessness was independently associated with being sputum smear positive (adjusted OR 1.8, 95\% CI 1.4-2.4), as was being aged under 45 years. Those born in South Asia were less likely to have infectious TB (than those born elsewhere). Among the 173 cases reporting drug misuse, no further characteristics were associated with infectiousness.

73 (19\%) out of 393 homeless patients had isoniazid resistant and 20 (5\%) out of 393 had MDR-TB. Homelessness was an independent risk factor for isoniazid (adjusted OR 1.9, 95\% CI 1.4-2.6) and MDR disease (adjusted OR 2.9, 95\% CI 1.6-5.2), while problem drug use was associated with isoniazid resistance. Being born in East Europe or East Asia, and a previous history of TB was also associated with drug resistance. Recent migrants were more likely to be MDR, while patients aged 65 years or more were less likely to have drug resistance.

Almost half of all homeless patients were non-adherent (258 out of 550), and 303 (72\%) out of (420) completed treatment. Homelessness was associated with non-adherence (adjusted OR 10.2, 95\% CI 7.9-13.2) and not completing treatment (adjusted OR 2.6, 95\% CI 2.0-3.3). The other social risk factors were also independently associated with non-adherence, as was young age (under 25 years), pulmonary disease, a previous history of TB and being born in Central and West Europe.

Other characteristics associated with not completing treatment were being aged under 25 years or older than 54, male, born in East Europe, having pulmonary disease and being a recent migrant.

The increased risk of infectious TB among patients with social risk factors may relate to lung damage from smoking tobacco and/or crack cocaine, or delayed diagnosis [6, 7]. Risk of drug resistance for those born in East Europe or East Asia reflects the burden of drug-resistant TB in those areas. The increased risk of drug-resistant $\mathrm{TB}$ among homeless people and drug users, after controlling for country of birth and previous treatment, suggests transmission of drug-resistant disease in London, where homelessness is also a known risk factor for clustering [8].

All social risk factors were associated with non-adherence. Patients experiencing homelessness were most at risk (adjusted OR 10.2, 95\% CI 7.9-13.2). This increased with social complexity: 83\% of patients with four factors were non-adherent compared with $16 \%$ with one risk factor.

Homelessness was associated with not completing treatment. Males and those born in East Europe were also less likely to complete treatment, possibly due to under-reporting of social risk factors. Poor outcomes among recent migrants may reflect a preference to return to home countries for treatment, and among older patients the impact of co-morbidities.

TB patients with social risk factors have a disproportionate public health impact. Just $4 \%$ experienced homelessness but this was $16 \%$ of MDR and $36 \%$ of non-adherent patients. This supports UK guidance recommending assessing and supporting patient social risk factors, maintaining adequate staffing to support socially complex cases, and using cohort review as a quality assurance tool $[4,9]$. UK guidance for homeless people and drug users also recommends targeted TB case finding using mobile digital chest radiology, integrated screening and treatment for latent TB infection, hepatitis C and HIV, and enhanced case management through diagnosis and treatment [9].

It has been recognised that TB control efforts in low-incidence countries should focus in big urban centres [10]. The implementation of targeted approaches was reviewed in an international survey of TB-elimination practices in low incidence European countries, and followed by a consensus statement of the European Centre for Disease Prevention and Control TB in big cities working group. This detailed recommendations to improve early case finding, case holding and treatment completion, especially among vulnerable groups $[11,12]$. Despite the mostly low incidence, the economic cost of TB in the EU remains considerable (total costs of $€ 536890315$ accumulated in 2012) [13].

Limitations to our study include that surveillance likely underestimates the prevalence of social risk factors. We increased by $24 \%$ the proportion known as homeless after matching to a specialist outreach service database (445-550). Patients missing information were assumed to not have that factor, which may have weakened associations identified. Other missing information reduced the study power to identify risk factors for infectiousness (sputum smear missing for $22 \%$ of pulmonary patients) and drug resistance (susceptibility unknown for $42 \%$ of patients). Individual HIV status was unknown; however, co-infection estimates were low at approximately $4 \%$ of TB patients during this time period (personal communication January 2016; PHE National Infection Service). 
TABLE 1 Multivariable analysis of patient characteristics associated with infectiousness, drug resistance, non-adherence and poor outcomes

\begin{tabular}{|c|c|c|c|c|c|c|c|c|c|c|c|}
\hline \multirow[t]{2}{*}{ Patient characteristics } & \multirow[t]{2}{*}{ Total $\mathbf{n}$} & \multicolumn{2}{|c|}{ Sputum smear positive ${ }^{\#}$} & \multicolumn{2}{|c|}{ Isoniazid resistant" } & \multicolumn{2}{|c|}{ Multi-drug resistant" } & \multicolumn{2}{|c|}{ Non-adherent } & \multicolumn{2}{|c|}{ Did not complete $^{+}$} \\
\hline & & aOR $(95 \% \mathrm{CI})^{\S}$ & p-value & aOR $(95 \% \mathrm{CI})$ & p-value & aOR $(95 \% \mathrm{CI})$ & p-value & aOR $(95 \% \mathrm{CI})$ & $\mathrm{p}$-value & aOR $(95 \% \mathrm{CI})$ & p-value \\
\hline Patients n & 12908 & \multicolumn{2}{|l|}{2179} & \multicolumn{2}{|l|}{646} & \multicolumn{2}{|c|}{127} & \multicolumn{2}{|l|}{717} & \multirow{2}{*}{\multicolumn{2}{|c|}{1207}} \\
\hline \multicolumn{10}{|l|}{ Age years } & & \\
\hline $16-24$ & 2289 & $1.2(1.0-1.4)$ & 0.059 & $1.0(0.8-1.3)$ & 0.877 & $2.0(1.2-3.1)$ & 0.004 & $1.5(1.2-1.9)$ & 0.001 & $1.2(1.1-1.4)$ & 0.001 \\
\hline $25-34$ & 4200 & Ref & & Ref & & Ref & & Ref & & Ref & \\
\hline $35-44$ & 2512 & $0.8(0.7-1.0)$ & 0.051 & $1.1(0.8-1.4)$ & 0.598 & $0.9(0.5-1.6)$ & 0.702 & $0.8(0.6-1.1)$ & 0.183 & $0.9(0.8-1.1)$ & 0.408 \\
\hline $45-54$ & 1638 & $0.7(0.6-0.9)$ & 0.004 & $1.2(0.9-1.5)$ & 0.273 & $1.1(0.6-2.1)$ & 0.772 & $0.8(0.6-1.1)$ & 0.216 & $1.2(1.0-1.5)$ & 0.074 \\
\hline $55-64$ & 980 & $0.7(0.6-0.9)$ & 0.012 & $1.0(0.7-1.5)$ & 0.957 & $0.6(0.21 .8)$ & 0.383 & $0.7(0.5-1.1)$ & 0.158 & $1.4(1.1-1.7)$ & 0.013 \\
\hline$>65$ & 1289 & $0.6(0.5-0.7)$ & $<0.001$ & $0.6(0.4-0.8)$ & 0.005 & $0.1(0.01-0.8)$ & 0.030 & $0.5(0.3-0.7)$ & 0.001 & $2.6(2.2-3.2)$ & $<0.001$ \\
\hline Male & 7470 & & & & & & & & & $1.3(1.1-1.4)$ & 0.0006 \\
\hline \multicolumn{12}{|l|}{ World region of birth ${ }^{f}$} \\
\hline Central Europe & 317 & $1.9(1.4-2.5)^{\S}$ & $<0.001$ & $0.8(0.5-1.4)$ & 0.435 & $1.7(0.8-3.9)$ & 0.194 & $2.0(1.3-3.1)$ & 0.002 & $1.3(0.9-1.9)$ & 0.100 \\
\hline East Asia & 147 & $1.8(1.2-2.7)^{\S}$ & 0.008 & $1.9(1.0-3.4)$ & 0.045 & $3.7(1.5-9.1)$ & 0.004 & $1.6(0.8-3.3)$ & 0.182 & $1.4(0.9-2.3)$ & 0.154 \\
\hline East Europe & 84 & $2.1(1.3-3.6)^{\S}$ & 0.005 & $4.0(2.2-7.2)$ & $<0.001$ & $7.6(3.4-17.0)$ & $<0.001$ & $1.9(0.9-4.1)$ & 0.116 & $1.9(1.1-3.3)$ & 0.026 \\
\hline East Mediterranean & 97 & $1.2(0.6-2.5)^{\S}$ & 0.570 & & & & & $0.3(0.1-1.4)$ & 0.128 & $1.5(0.8-2.9)$ & 0.253 \\
\hline North Africa & 112 & $1.3(0.7-2.4)^{\S}$ & 0.450 & & & & & $0.7(0.3-1.9)$ & 0.504 & $0.5(0.2-1.1)$ & 0.072 \\
\hline $\begin{array}{l}\text { North America and } \\
\text { Oceania }\end{array}$ & 26 & $0.9(0.3-2.7)^{\S}$ & 0.811 & & & & & $0.9(0.1-7.0)$ & 0.926 & $1.1(0.3-3.6)$ & 0.931 \\
\hline South Asia & 5888 & Ref & & Ref & & Ref & & Ref & & Ref & \\
\hline South East Asia & 393 & $1.4(1.0-1.9)^{\S}$ & 0.032 & $1.1(0.7-1.8)$ & 0.738 & $1.0(0.3-2.7)$ & 0.93 & $1.6(1.0-2.6)$ & 0.077 & $1.1(0.8-1.5)$ & 0.690 \\
\hline $\begin{array}{c}\text { South, Central America } \\
\text { and the Caribbean }\end{array}$ & 297 & $2.3(1.7-3.2)^{\S}$ & $<0.001$ & $0.8(0.4-1.5)$ & 0.523 & & & $1.6(0.9-2.8)$ & 0.081 & $0.9(0.6-1.4)$ & 0.651 \\
\hline Sub-Saharan Africa & 3023 & $1.2(1.1-1.4)^{\S}$ & 0.004 & $0.8(0.6-1.0)$ & 0.068 & $0.6(0.4-1.0)$ & 0.045 & $1.0(0.8-1.2)$ & 0.74 & $0.7(0.6-0.9)$ & $<0.001$ \\
\hline West Europe ${ }^{\# \#}$ & 1948 & $1.9(1.7-2.3)^{\S}$ & $<0.001$ & $1.1(0.9-1.4)$ & 0.318 & $0.6(0.3-1.1)$ & 0.073 & $1.3(1.0-1.7)$ & 0.046 & $1.1(0.9-1.3)$ & 0.248 \\
\hline Recent migrant $(<2 \text { years })^{\#}$ & 1981 & & & & & $1.7(1.1-2.8)$ & 0.023 & & & $1.5(1.3-1.8)$ & $<0.0001$ \\
\hline Previous TB & 772 & & & $1.5(1.1-2.2)$ & 0.026 & $4.4(2.6-7.5)$ & $<0.001$ & $1.7(1.2-2.3)$ & 0.0029 & & \\
\hline Pulmonary & 6184 & & & & & & & $1.7(1.4-2.0)$ & $<0.0001$ & $1.2(1.1-1.4)$ & 0.0013 \\
\hline \multicolumn{12}{|l|}{ Social risk factors } \\
\hline Problem drug use & 436 & & & $2.4(1.7-3.3)$ & $<0.0001$ & & & $3.0(2.2-4.1)$ & $<0.0001$ & & \\
\hline Alcohol & 581 & $1.4(1.1-1.8)^{\S}$ & 0.0140 & & & & & $2.9(2.2-3.9)$ & $<0.0001$ & & \\
\hline Prison & 349 & & & & & & & $2.3(1.6-3.2)$ & $<0.0001$ & & \\
\hline Homelessness & 550 & $1.8(1.4-2.4)^{\S}$ & $<0.0001$ & $1.9(1.4-2.6)$ & 0.0003 & $2.9(1.6-5.2)$ & $<0.001$ & $10.2(7.9-13.2)$ & $<0.0001$ & $2.6(2.0-3.3)$ & $<0.0001$ \\
\hline
\end{tabular}

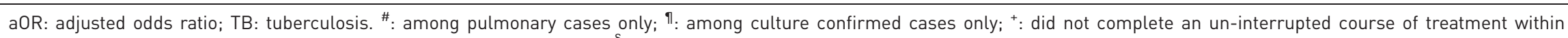
12 months if rifampicin sensitive, and 24 months if rifampicin resistant; ${ }^{\S}$ : among patients with problem drug use, no further characteristics were associated with sputum smear positive disease; ${ }^{f}$ : world region of birth is based on UK Enhanced TB Surveillance classification, based on country of birth; ${ }^{\# \#}$ : $76 \%$ of patients from West Europe were born in the UK. 
Our study confirms that TB patients in London with social risk factors are more likely to be infectious, drug resistant, and not complete treatment, and reveals homelessness as an independent risk factor for MDR disease. This convergence of clinical and social complexity presents an immense challenge and underlines the need for investment in specialist outreach services to tackle $\mathrm{TB}$ among vulnerable and medically under-served populations. We welcome the Collaborative Tuberculosis Strategy for England 2015-2020 which committed new investment to tackling TB in under-served populations [14].

$\boldsymbol{0}$ @ERSpublications

Clinically complex TB among socially vulnerable groups in London exemplifies the challenge of 21st century TB control http://ow.ly/DUzR302h1mD

Charlotte Anderson ${ }^{1}$, Sarah R. Anderson ${ }^{2}$, Helen Maguire ${ }^{1,3}$, Andrew C. Hayward ${ }^{3}$ and Alistair Story ${ }^{4}$

${ }^{1}$ Field Epidemiology Service - South East and London, Public Health England, London, UK. ${ }^{2}$ North West London Health Protection Team, Public Health England, London, UK. ${ }^{3}$ UCL Department of Infectious Disease Informatics, University College London, London, UK. ${ }^{4}$ Find\&Treat, University College Hospitals NHS Foundation Trust, London, UK.

Correspondence: Charlotte Anderson, Public Health England Field Epidemiology Service, Zone C, Floor 3 Skipton House, 80 London Road, London SE1 6LH. E-mail: charlotte.anderson@phe.gov.uk

Received: Feb 012016 | Accepted after revision: July 112016 | First published online: Sept 012016

This article has supplementary material available from erj.ersjournals.com

Conflict of interest: None declared.

Acknowledgements: We would like to thank London TB clinic staff and patients for providing information to the London TB surveillance system; Find\&Treat staff for providing information on patients referred to their service; PHE Field Epidemiology Service team for supporting the London TB surveillance system; PHE National Mycobaterium Reference Laboratory for providing information on culture and drug susceptibility; PHE National Infection Service TB section for linking case reports to laboratory data.

Authors' contributions: Charlotte Anderson carried out the analysis and wrote the first draft of the manuscript. Charlotte Anderson and Alistair Story developed the analysis plan. All authors contributed to interpretation of the findings, drafting of paper, approval for publication and are accountable for the quality and integrity of the work.

\section{References}

1 Lönnroth K, Migliori GB, Abubakar I, et al. Towards tuberculosis elimination: an action framework for low-incidence countries. Eur Respir J 2015; 45: 928-952.

2 de Vries G, Aldridge RW, Caylà JA, et al. The Tuberculosis in European Union Big Cities Working Group. Epidemiology of tuberculosis in big cities of the European Union and European Economic Area countries. Euro Surveill 2014; 19: 20726.

3 Jit M, Stagg HR, Aldridge RW, et al. Dedicated outreach service for hard to reach patients with tuberculosis in London: observational study and economic evaluation. BMJ 2011; 343: d5376.

4 Royal College of Nursing. Tuberculosis Case Management and Cohort Review. London, March 2012. Available from www.rcn.org.uk/_data/assets/pdf_file/0010/439129/004204.pdf Date last accessed: November, 2015.

5 World Health Organization, Geneva. Tuberculosis factsheet March 2016. Available from www.who.int/ mediacentre/factsheets/fs104/en/ Date last accessed: March, 2016.

6 Ward HA, Marciniuk DD, Pahwa P, et al. Extent of pulmonary tuberculosis in patients diagnosed by active compared to passive case finding. Int J Tuberc Lung Dis 2004; 8: 593-597.

7 Story A, Bothamley G, Hayward A. Crack cocaine and infectious tuberculosis. Emerg Infect Dis 2008; 14: 1466-1469.

8 Love J, Sonnenberg P, Glynn JR, et al. Molecular epidemiology of tuberculosis in England, 1998. Int J Tuberc Lung Dis 2009; 13: 201-207.

9 National Institute for Health and Clinical Care Excellence. NICE Guidance PH37, Identifying and managing tuberculosis among hard to reach groups. 2012. Available from http://guidance.nice.org.uk/PH37 Date last accessed: November, 2015.

10 Sotir MJ, Parrott P, Metchock B, et al. Tuberculosis in the inner city: impact of a continuing epidemic in the 1990s. Clin Infect Dis 1999; 29: 1138-1144.

11 D'Ambrosio L, Dara M, Tadolini M, et al. Tuberculosis elimination: theory and practice in Europe. Eur Respir J 2014; 43: 1410-1420.

12 Van Hest NA, Aldridge RW, de Vries G, et al. Tuberculosis control in big cities and urban risk groups in the European Union: a consensus statement. Euro Surveill 2014; 19.

13 Diel R, Vandeputte J, de Vries G, et al. Costs of tuberculosis disease in the European Union: a systematic analysis and cost calculation. Eur Respir J 2014; 43: 554-565.

14 Public Health England and NHS England. Collaborative Tuberculosis Strategy for England 2015 to 2020. London January 2015. www.gov.uk/government/uploads/system/uploads/attachment_data/file/403231/Collaborative_TB_ Strategy_for_England_2015_2020_.pdf Date last accessed: November, 2015. 